Rodríguez Flores, E.A. y Sánchez Trujillo, Ma .A. (2018). Síndrome de Burnout y variables sociodemográficas en docentes de una universidad privada de Lima. Revista de Investigación Educativa, 36(2), 401-419.

DOI: http://dx.doi.org/10.6018/rie.36.2.282661

\title{
Síndrome de Burnout y variables sociodemográficas en docentes de una universidad privada de Lima
}

\section{Burnout Syndrome and Socio-demographic Variables in Teachers from a Private University in Lima}

\author{
Eduar Antonio Rodríguez Flores y María de los Ángeles Sánchez Trujillo \\ Departamento de Humanidades, Universidad Peruana de Ciencias Aplicadas, Lima, Perú.
}

\begin{abstract}
Resumen
El objetivo principal de la presente investigación fue determinar las diferencias entre los componentes del síndrome de burnout en docentes de una universidad privada de Lima según variables sociodemográficas. Para ello, se utilizó un diseño de investigación descriptivo comparativo. También, debido a que no se aplicó muestreo, se abarcó a una población total compuesta por 260 docentes de Humanidades de dicha institución educativa, quienes dictan cursos de Lenguaje, de Investigación, Ética y Ciudadanía, y Taller de Creatividad. Además, se utilizó como instrumentos de medición la adaptación del Inventario Burnout de Maslach y una ficha sociodemográfica para docentes universitarios. A partir del procesamiento y análisis estadístico de los datos obtenidos, se halló lo siguiente: existen diferencias significativas de cansancio emocional según sexo y turno de dictado; asimismo, de realización personal según edad, estado civil, especialidad, máximo nivel de estudios alcanzado y años de experiencia docente; entre otros resultados. Además, se corroboró o refutó algunos resultados de investigaciones previas, especialmente, en relación con las variables sexo, edad, estado civil, nivel de estudios y turno de dictado. También, a partir de este trabajo, se identificó que el lugar de estudios de pregrado, la especialidad y los años de experiencia, aspectos no estudiados a profundidad en los antecedentes de estudio, podrían resultar variables asociadas a algunos
\end{abstract}

Correspondencia: María de los Ángeles Sánchez Trujillo, pchemsan@upc.edu.pe, Universidad Peruana de Ciencias Aplicadas, Perú. 
componentes del burnout. Finalmente, además de las diferencias significativas mencionadas, se concluyó que existen niveles medios de cansancio emocional, despersonalización, distorsión del entorno laboral, realización personal y realización laboral en los docentes que conformaron la muestra de estudio.

Palabras clave: estrés; gestión del centro de enseñanza; profesión docente; condiciones de trabajo del profesor; psicología social; salud mental.

\begin{abstract}
The main objective of this research was to determine the differences among the components of burnout in professors from a private university in Lima according to socio-demographic variables. With this aim in mind, a comparative descriptive research design was used. Also, a specific sample quota was not applied. We collected a total population composed of 260 professors of humanities at said educational institution who teach the following courses: Language, Research, Ethics and Citizenship and Creativity Workshop. In addition, we used the Maslach Burnout Inventory Adaptation and a socio-demographic record handout for university professors as measuring instruments. Finally, from the processing and statistical analysis of the data obtained, we found the following: there are significant differences in the levels of emotional exhaustion by sex and class schedule; also, personal fulfillment according to age, marital status, specialty, highest level of educational attainment and years of teaching experience, among other results. Some results of previous research were corroborated or refuted, especially in relation to the variables sex, age, marital status, level of education and class schedule. Also, from this research, it was identified that the place of undergraduate studies, specialty and years of teaching experience, aspects not studied in depth in the previous studies, could be variables associated with some components of burnout. Finally, in addition to the significant differences mentioned, it was concluded that there are average levels of emotional exhaustion, depersonalization, distortion of the work environment, personal and professional fulfillment in the teachers who formed the study sample.
\end{abstract}

Keywords: stress; school management; teaching profession; working conditions of teachers; social psychology; mental health.

\title{
Introducción
}

El presente artículo se basa en los resultados que fueron producto del desarrollo de la tesis de maestría en Educación con mención en Gestión Educativa que realizaron los autores. El trabajo se orientó a indagar acerca del síndrome de burnout y las variables sociodemográficas en docentes de una universidad privada de Lima.

El síndrome de burnout se manifiesta como una reacción física y psicológica negativa luego de una exposición a situaciones de estrés dentro del contexto laboral (Maslach, Schaufeli \& Leiter, 2001). Cabe resaltar que, en sus primeras investigaciones, Maslach y Jackson (1986) definieron los tres componentes que constituyen el fenómeno del burnout: cansancio emocional, despersonalización y baja realización personal. Según Gil-Monte y Peiró (1999), así como Benfica y Barbosa (2015), es posible afirmar que la necesidad de estudiar este fenómeno se vincula con la preocupación de las organizaciones por la calidad de vida laboral de sus empleados no solo por su bienestar personal, sino 
por los efectos que producen en la institución (Cárdenas, Méndez \& González, 2014; Cattaneo \& Mandolesi, 2013; Fernández, Ruiz, Toledo \& Conchas, 2015; Jenaabadi, Azizi, Saeidi, Haghi \& Hojatinasab, 2016). Incluso, se podría producir la generación de interrelaciones dañinas, el absentismo y, en términos extremos, el abandono de la organización, según Rabasa, Figuereido- Ferraz, Gil- Monte y Llorca- Pellicer (2016). Por otro lado, como mencionan Agudo (2006) y Tenti (2007), la docencia es considerada como una de las profesiones más propensas a padecer problemas de salud mental, entre los que se incluye el burnout.

En Perú, hay una despreocupación por parte del Estado en brindar condiciones laborales apropiadas a los profesores. En las instituciones de educación superior, ello puede agravarse, debido a la gran cantidad de profesores pertenecientes a distintas áreas de especialización. Si a esto se suma la frecuente rotación docente y la gran cantidad de trabajo administrativo que deben realizar, además de su labor de enseñanza, entonces podrían estar más propensos a padecer burnout.

El objetivo de la presente investigación es determinar las diferencias entre los componentes del síndrome de burnout según determinadas variables sociodemográficas en docentes universitarios de Lima. Al respecto las hipótesis planteadas son, por un lado, que existen niveles altos de cansancio emocional, despersonalización y distorsión del entorno laboral, y niveles bajos de realización personal y realización laboral en docentes de una universidad privada de Lima. Por otro lado, se plantea que existen diferencias significativas entre los componentes del síndrome de burnout según sexo, edad, estado civil, lugar de estudios de pregrado, especialidad, máximo nivel de estudios de pregrado, años de experiencia docente y turno de dictado.

Cabe resaltar que la importancia de esta indagación radica en que, en el país, no existe bibliografía suficiente en relación con el tema y menos aún estudios realizados con población docente de instituciones de educación superior. Pese a ello, hemos tenido en cuenta algunos estudios que han permitido corroborar los resultados o refutarlos. Respecto de la variable sexo, existen estudios que concluyen que las mujeres se sienten más cansadas a nivel emocional respecto de los hombres, tales como los realizados por Arias y Jiménez (2013), Asitimbay (2015), Manassero, Fornés, Fernández, Vásquez y Ferrer (1995), Martínez (2015), Montejo (2014), Oliver (1993), Santiago, Otero- López, Castro y Villadefrancos (2008), Tifner, Martín, Albanesi y De Bortoli (2006) y Vidal (2013).

Por otro lado, a partir de la investigación ejecutada por Zaldívar, Estrada, Qui, Moncada y Aguilar (2013) en profesores universitarios de la Universidad Autónoma de Sinaloa, se concluyó que los docentes más jóvenes suelen presentar un mayor nivel de burnout. Por otra parte, según los estudios efectuados por Manassero et al (1995), los docentes con mayor edad se sienten más realizados a nivel personal. Un aspecto ligado a la edad es el tiempo de servicio. Al respecto, Ponce, Bulnes, Aliaga, Atalaya y Huerta (2014) realizaron un estudio con profesores de la Universidad Nacional Mayor de San Marcos en Perú y concluyeron que, mientras mayor es el tiempo de servicio, es menor el cansancio personal y la despersonalización, y mayor realización personal. No obstante, de acuerdo con los resultados de las investigaciones realizadas por Boada, De Diego y Agulló (2004), Díaz, López y Varela (2010) y Esteras, Chorot y Sandín (2014), ni la edad ni la antigüedad guardan una relación directa con la manifestación del síndrome de burnout. 
En cuanto al estado civil, Maslach y Jackson (1986) la consideraron como una variable relevante. Autores como Silva, García, González y Ratto (2015) afirman que los divorciados estarían más propensos a sentirse realizados a nivel personal.

El estudio de Ibáñez et al. (2012) también resulta relevante, pues identificaron que los profesores que pertenecen a estratos altos suelen sentirse más cansados a nivel emocional, posiblemente, por la poca tolerancia a la frustración que suelen manifestar. Adicionalmente, reconocieron que aquellos que trabajan en más de una jornada presentan un cansancio más elevado.

Cabe señalar que la especialidad del docente no es una variable que haya sido estudiada en las investigaciones sobre el burnout. Sólo Manassero et al. (1995), a partir de un estudio realizado en un centro de Educación Básica Regular, señalaron que los docentes que no han estudiado Educación podrían manifestar mayores síntomas de burnout en la medida en que perciban que sus labores no les ayudan a desarrollarse profesionalmente.

Finalmente, a partir del estudio realizado sobre el tema, Tifner et al. (2006) plantearon que el estatus percibido por los profesores influye en su bienestar emocional. Por tanto, su categoría docente y sueldo podrían ser variables asociadas a algunos componentes de burnout.

Cabe señalar que se consideró justificable realizar la exploración, dado que no es posible mejorar la gestión de una institución educativa sin atender a las condiciones de salud de la plana docente, uno de los pilares de la formación de los estudiantes.

\section{Método}

\section{Diseño}

La investigación obedece a un diseño descriptivo comparativo (Sánchez \& Reyes, 2015), puesto que, sobre la base de descripciones simples respecto de un mismo objeto de estudio, se establecieron comparaciones entre los datos obtenidos. En este caso, se determinaron las diferencias que existen entre los componentes del síndrome de burnout de docentes de una universidad privada de Lima según variables sociodemográficas.

\section{Población y Muestra}

La población estuvo conformada por los docentes de Humanidades que dictan cursos de formación general y que laboran en la universidad en donde se realizó la investigación (260 en total). Se ha optado por este universo de profesores, debido a que, según Manassero et al. (1995), las personas que se encuentran más expuestas al síndrome de burnout son aquellas que no ejercen su profesión directamente, lo cual se acentúa en el caso de los profesores. Precisamente, ello ocurre con los docentes de Humanidades que dictan cursos de instrucción general en una institución orientada, específicamente, a la formación en Ciencias. De igual modo, cabe señalar que se trabajó con una muestra exhaustiva (Hernández, Fernández \& Baptista, 2014). Del total de docentes, 195 provienen de Lima, 60 de provincia y 5 son extranjeros. Además, la media de edad correspondía a docentes entre 31 y 40 años. Asimismo, se identificaron 113 docentes del sexo femenino y 147, del sexo masculino. 


\section{Instrumentos}

En primer lugar, se empleó una adaptación del Inventario Burnout de Maslach -denominado Maslach Burnout Inventory (MBI) en su versión original-, el cual fue elaborado por Cristina Maslach y Susan Jackson, y fue publicado en dos versiones: la de 1981 y la de 1986. El propósito del instrumento es evaluar los tres componentes básicos del síndrome de burnout (cansancio emocional, despersonalización y realización personal). De igual modo, el MBI consta de 22 ítems, los cuales están distribuidos desigualmente en relación con los componentes del síndrome. Por ejemplo, nueve preguntas están destinadas a evaluar el cansancio emocional; cinco la despersonalización; y ocho la realización personal. Asimismo, cada ítem del instrumento posee una escala de frecuencia tipo Likert que oscila entre de 0 (nunca) y 6 (todos los días). Además, se declara que una persona padece de síndrome de burnout cuando obtiene altos niveles en los dos primeros componentes y bajos en el tercero.

Para la presente investigación, a partir del Inventario Burnout de Maslach empleado por Farfán (2009) en su tesis, se realizó una nueva validación de dicho instrumento mediante un análisis factorial de los ítems. Para ello, se realizó una prueba piloto en la que participaron 200 docentes pertenecientes a la población de estudio. Producto de dicho procedimiento estadístico con la herramienta SPSS 22, en el que se cumplieron los presupuestos estadísticos que declaran válido el procedimiento (determinante: $.000<2$; prueba Kaiser-Meyer-Olkin: .823 > .70; y prueba de esfericidad de Barlett: .000 <.05), el Inventario Burnout de Maslach se reagrupó en seis componentes con sus respectivos ítems: cansancio emocional, realización personal, realización laboral, distorsión del entorno laboral, despersonalización y apatía. Es decir, se agregaron los componentes "realización laboral", "distorsión del entorno laboral" y "apatía", que no contemplaban el inventario original ni la validación realizada por Farfán (2009).

Después de realizar el análisis factorial y de identificar la formación de nuevos componentes, los cuales fueron renominados en función de la lógica de cada conjunto, fue necesario aplicar nuevamente pruebas de fiabilidad para corroborar la consistencia interna de las nuevas dimensiones. Los resultados permitieron identificar que todos los componentes obtuvieron coeficientes de alfa de Cronbach superiores a .70, salvo en el caso del componente "apatía", el cual se decidió eliminar (tres ítems), puesto que su fiabilidad era de $.558<.70$, y en el de "realización personal", cuya fiabilidad pasaba del $.678<.70$ al $.770>.70$ al eliminar un ítem. En ese sentido, el Inventario quedó reducido de 22 a 18 ítems distribuidos en cinco componentes y con el mismo rango de respuesta de 0 a 6 por ítem, como se consignaba en el original.

El baremo que Maslach y Jackson (1986) emplearon para clasificar la variabilidad de resultados respecto de los componentes del síndrome de burnout fue dividido en tres partes. Partiendo de ello, un nivel bajo está representado por los resultados ubicados en el tercio inferior (centiles del 1 a 33); un nivel medio, por resultados entre centiles 34 a 66; y un nivel alto, por puntuaciones del tercio superior (centiles 67 a 99). A partir de esto, para declarar que una persona padece de síndrome de burnout, es necesario que obtenga altos puntajes de cansancio emocional y despersonalización, y bajos de realización personal. 
Para efectos de la presente investigación, producto del análisis factorial para validación de constructo, se reclasificaron los ítems del instrumento en cinco dimensiones: cansancio emocional, despersonalización, distorsión del entorno laboral, realización personal y realización laboral. Asimismo, a partir de los resultados obtenidos, se realizó una nueva identificación del baremo siguiendo la pauta original de Maslach y Jackson. Para ello, mediante el programa SPSS, se aplicó la sumatoria de ítems de los nuevos componentes y una prueba de frecuencias. De este modo, para declarar el síndrome de burnout, siguiendo la lógica del instrumento original, los resultados, en las primeras tres dimensiones, debían corresponder al tercio superior (centiles 67 a 99) y, en las dos últimas, al tercio inferior (centiles 1 a 33).

En segundo lugar, se empleó una ficha sociodemográfica destinada a ser aplicadas a docentes universitarios, la cual fue elaborada por los autores. El instrumento está compuesto por 8 variables, las cuales son las siguientes:

1. Sexo

2. Edad

3. Estado civil

4. Lugar de estudios de pregrado

5. Especialidad

6. Máximo nivel de estudios alcanzado

7. Años de experiencia docente

8. Turno de dictado

\section{Procedimiento de recojo y análisis de datos}

Para el recojo de información, se aprovecharon las reuniones de coordinación de los cursos de la universidad para suministrar los instrumentos. En principio, se realizó la presentación del equipo de investigación, previa anuencia del coordinador de la asignatura respectiva. Luego, se explicó el objetivo, la justificación y la importancia de la participación de los profesores. Asimismo, se solicitó la firma del consentimiento informado en caso de que los docentes acepten ser parte de la investigación, y se enfatizó en el anonimato y la discreción respecto de los datos de los participantes. Posteriormente, se repartió el cuadernillo que contiene la ficha sociodemográfica y el Inventario adaptado Burnout de Maslach, y también se realizaron las aclaraciones necesarias para la correcta respuesta a los ítems planteados.

Luego de finalizada la aplicación de los instrumentos, se efectuó el análisis estadístico de la información encontrada. Para ello, se aplicaron pruebas descriptivas para identificar las medias y los coeficientes de variación, pruebas de normalidad (ShapiroWilk para grupos de 50 sujetos o menos, y Kolmogorov-Smirnov para grupos de más de 50) para definir si debía procederse con paramétricas (Análisis de varianza ANOVA para más de dos grupos independiente) o no paramétricas (" $U$ " de Mann-Whitney para grupos independientes o " $\mathrm{H}$ " de Krustal-Walls para más de dos grupos independientes). En todos los casos, dichos procedimientos se realizaron mediante el empleo de la herramienta estadística SPSS 22. 


\section{Resultados}

Luego del recojo de información mediante los instrumentos de medición considerados para el estudio, se procedió a realizar el procesamiento de la data con el programa estadístico SPSS 22.

En la tabla 1, se muestran los resultados obtenidos en las categorías correspondientes a las variables sociodemográficas.

Tabla 1

Resultados obtenidos en las categorías de variables sociodemográficas

\begin{tabular}{ll}
\hline \multicolumn{1}{c}{ Variable sociodemográfica } & Resultados obtenidos según categorías \\
\hline Sexo & Femenino: 113 \\
& Masculino: 147 \\
\hline Edad & Entre 20 y 30 años: 66 \\
& Entre 31 y 40 años: 130 \\
& Entre 41 y 50 años: 54 \\
& Entre 51 y 60 años: 10 \\
& De 61 años a más: 0 \\
\hline Estado civil & Soltero: 138 \\
& Casado: 77 \\
& Viudo: 0 \\
& Divorciado: 13 \\
& Separado: 11 \\
& Conviviente: 21 \\
\hline Lugar de estudios de pregrado & Universidad estatal: 117 \\
& Universidad privada: 143 \\
\hline Especialidad & Educación: 52 \\
& Literatura: 91 \\
& Lingüística: 37 \\
& Filosofía: 34 \\
& Historia: 6 \\
& Antropología: 6 \\
& Sociología: 5 \\
& Comunicaciones: 19 \\
& Psicología: 7 \\
& Administración: 3 \\
\hline Máximo nivel de estudios alcanzado & Bachiller: 63 \\
& Licenciado(a): 90 \\
& Magíster: 94 \\
& Doctor(a): 13 \\
& \\
& \\
& \\
& \\
& \\
& \\
& \\
& \\
& \\
& \\
& \\
& \\
& \\
& \\
& \\
& \\
&
\end{tabular}




\begin{tabular}{ll}
\hline Años de experiencia docente & Entre 1 y 5 años: 82 \\
& Entre 6 y 10 años: 78 \\
& Entre 11 y 15 años: 46 \\
& Entre 16 y 20 años: 32 \\
& Entre 21 y 25 años: 15 \\
& Entre 26 y 30 años: 7 \\
& Entre 31 y 35 años: 0 \\
\hline Turno de dictado & Mañana: 40 \\
& Tarde: 19 \\
& Noche: 2 \\
& Mañana y tarde: 101 \\
& Tarde y noche: 21 \\
& Mañana y noche: 16 \\
& Mañana, tarde y noche: 61 \\
\hline
\end{tabular}

A continuación, se brindará información acerca de los niveles evidenciados por los docentes en los componentes de burnout, así como las diferencias significativas evidenciadas entre estos según variables sociodemográficas. Cabe mencionar que, en algunas variables de tres a más categorías, se ha identificado que las que corresponden a cansancio emocional tienen una distribución normal, mientras que las de los demás componentes del síndrome de burnout, una de tipo anormal. Debido a ello, en tales casos, el análisis se realizó por separado, puesto que se emplearon dos tipos de pruebas: ANOVA para comparar cansancio emocional y Kruskal-Wallis para comparar los demás componentes. Esto ocurre en las variables referidas a edad, estado civil, especialidad, años de experiencia docente y turno de dictado, por lo cual, en la presentación de cada uno de estos resultados, se están mostrando dos tablas: una, acerca del cansancio emocional; y la otra, sobre los demás componentes.

En la tabla 2, se aprecian las medias de los componentes del síndrome de burnout, las cuales, si se contrastan con el baremo, indican lo siguiente: existen niveles medios de cansancio emocional, despersonalización (cercano al alto: $1.95<2.00$ ), distorsión del entorno laboral, realización personal y realización laboral, por lo cual se rechaza la hipótesis alterna de que existen niveles altos de cansancio emocional, despersonalización y distorsión del entorno laboral, y niveles bajos de realización personal y realización laboral en docentes de una universidad privada de Lima. Asimismo, se ha identificado que los coeficientes de variación del cansancio emocional, despersonalización y distorsión del entorno laboral son mayores de 33 \% (mínimo para declarar dispersión para muestras mayores de 100 participantes), lo cual evidencia que las medias no representan adecuadamente la muestra. Cabe señalar que los resultados mostrados en la tabla corresponden con los obtenidos en el baremo, a partir de la sumatoria de los puntajes de 0 a 6 alcanzados en las preguntas que competen a cada componente del burnout. Así, el puntaje máximo obtenido para declarar el nivel de cada componente depende de la sumatoria de los puntajes logrados en las preguntas respectivas. 
Tabla 2

Estadísticos descriptivos de los componentes del síndrome de burnout

\begin{tabular}{lcccccc}
\hline & N & Mínimo & Máximo & Media & $\begin{array}{c}\text { Desviación } \\
\text { estándar }\end{array}$ & $\begin{array}{c}\text { Coeficiente } \\
\text { de variación }\end{array}$ \\
\hline Cansancio emocional & 260 & 0 & 24 & 12.75 & 5.921 & 46.46 \\
Despersonalización & 260 & 0 & 12 & 1.95 & 2.879 & 147.95 \\
Distorsión del entorno laboral & 260 & 0 & 30 & 7.26 & 6.039 & 83.17 \\
Realización personal & 260 & 2 & 20 & 15.11 & 2.933 & 19.41 \\
Realización laboral & 260 & 1 & 24 & 19.45 & 4.192 & 21.55 \\
N válido (por lista) & 260 & & & & & \\
\hline
\end{tabular}

En la tabla 3, que concierne a la variable sexo, se evidencia que todas las significancias, salvo en cansancio emocional y realización laboral, son mayores a .05. Por tanto, no existen diferencias significativas entre los componentes despersonalización, distorsión del entorno laboral y realización personal según sexo, y existen diferencias significativas entre los componentes cansancio emocional y realización laboral según sexo.

Tabla 3

Estadísticos de prueba U de Mann-Whitney para componentes del síndrome de burnout según sexo

\begin{tabular}{lccccc}
\hline & $\begin{array}{c}\text { Cansancio } \\
\text { emocional }\end{array}$ & $\begin{array}{c}\text { Despersona- } \\
\text { lización }\end{array}$ & $\begin{array}{c}\text { Distorsión } \\
\text { del entorno } \\
\text { laboral }\end{array}$ & $\begin{array}{c}\text { Realización } \\
\text { personal }\end{array}$ & $\begin{array}{c}\text { Realización } \\
\text { laboral }\end{array}$ \\
\hline U de Mann-Whitney & 7047.500 & 7351.500 & 7977.000 & 8191.500 & 7040.000 \\
W de Wilcoxon & 17925.500 & 13792.500 & 18855.000 & 19069.500 & 13481.000 \\
Z & -2.096 & -1.727 & -.548 & -.193 & -2.119 \\
Sig. asintótica (bilateral) & .036 & .084 & .584 & .847 & .034 \\
\hline
\end{tabular}

En la tabla 4, que concierne a la variable edad, se evidencia que la significancia es mayor a .05. Por lo tanto, no existen diferencias significativas de cansancio emocional según edad.

Tabla 4

Estadísticos de prueba ANOVA para cansancio emocional según edad

\begin{tabular}{lccccc}
\hline & $\begin{array}{c}\text { Suma de } \\
\text { cuadrados }\end{array}$ & Gl & $\begin{array}{c}\text { Media } \\
\text { cuadrática }\end{array}$ & F & Sig. \\
\hline Entre grupos & 97.276 & 3 & 32.425 & .924 & .430 \\
Dentro de grupos & 8983.970 & 256 & 35.094 & & \\
Total & 9081.246 & 259 & & & \\
\hline
\end{tabular}


En la tabla 5, que concierne también a la variable edad, se evidencia que todas las significancias, salvo en realización personal, son mayores a .05. Por lo tanto, no existen diferencias significativas entre los componentes despersonalización, distorsión del entorno laboral y realización laboral según edad, y existen diferencias significativas de realización personal según edad.

Tabla 5

Estadísticos de prueba Kruskal-Wallis para cuatro componentes del síndrome de burnout según edad

\begin{tabular}{lcccc}
\hline & Despersonalización & $\begin{array}{c}\text { Distorsión del } \\
\text { entorno laboral }\end{array}$ & $\begin{array}{c}\text { Realización } \\
\text { personal }\end{array}$ & $\begin{array}{c}\text { Realización } \\
\text { laboral }\end{array}$ \\
\hline Chi-cuadrado & 4.253 & 1.299 & 20.168 & 5.233 \\
Gl & 3 & 3 & 3 & 3 \\
Sig. asintótica & .235 & .729 & .000 & .155 \\
\hline
\end{tabular}

En la tabla 6, que concierne a la variable estado civil, se evidencia que la significancia es mayor a .05. Por lo tanto, no existen diferencias significativas de cansancio emocional según estado civil.

Tabla 6

Estadísticos de prueba ANOVA para cansancio emocional según estado civil

\begin{tabular}{lccccc}
\hline & Suma de cuadrados & Gl & Media cuadrática & F & Sig. \\
\hline Entre grupos & 261.946 & 4 & 65.486 & 1.893 & .112 \\
\hline Dentro de grupos & 8819.300 & 255 & 34.585 & & \\
\hline Total & 9081.246 & 259 & & & \\
\hline
\end{tabular}

En la tabla 7, que concierne también a la variable estado civil, se evidencia que todas las significancias, salvo en realización personal, son mayores a .05. Por lo tanto, no existen diferencias significativas entre los componentes despersonalización, distorsión del entorno laboral y realización laboral según estado civil, y existen diferencias significativas de realización personal según estado civil.

Tabla 7

Estadísticos de prueba Kruskal-Wallis para cuatro componentes del síndrome de burnout según estado civil

\begin{tabular}{lcccc}
\hline & Despersonalización & $\begin{array}{c}\text { Distorsión del } \\
\text { entorno laboral }\end{array}$ & $\begin{array}{c}\text { Realización } \\
\text { personal }\end{array}$ & $\begin{array}{c}\text { Realización } \\
\text { laboral }\end{array}$ \\
\hline Chi-cuadrado & 5.167 & 1.740 & 17.064 & 6.843 \\
Gl & 3 & 3 & 3 & 3 \\
Sig. asintótica & .160 & .628 & .001 & .077 \\
\hline
\end{tabular}


En la tabla 8, que concierne a la variable lugar de estudios de pregrado, se evidencia que todas las significancias, salvo en distorsión del entorno laboral, son mayores a .05. Por lo tanto, no existen diferencias significativas entre los componentes cansancio emocional, despersonalización, realización personal y realización laboral según lugar de estudios de pregrado, y existen diferencias significativas de distorsión del entorno laboral según lugar de estudios de pregrado.

Tabla 8

Estadísticos de prueba U de Mann-Whitney para componentes del síndrome de burnout según lugar de estudios de pregrado

\begin{tabular}{lccccc}
\hline & $\begin{array}{c}\text { Cansancio } \\
\text { emocional }\end{array}$ & $\begin{array}{c}\text { Despersona- } \\
\text { lización }\end{array}$ & $\begin{array}{c}\text { Distorsión } \\
\text { del entorno } \\
\text { laboral }\end{array}$ & $\begin{array}{c}\text { Realización } \\
\text { personal }\end{array}$ & $\begin{array}{c}\text { Realización } \\
\text { laboral }\end{array}$ \\
\hline U de Mann-Whitney & 7847.500 & 7557.500 & 7096.500 & 8336.000 & 8116.500 \\
W de Wilcoxon & 14750.500 & 14460.500 & 13999.500 & 15239.000 & 18412.500 \\
Z & -.860 & -1.457 & -2.108 & -.050 & -.415 \\
$\begin{array}{l}\text { Sig. asintótica } \\
\text { (bilateral) }\end{array}$ & .390 & .145 & .035 & .960 & .678 \\
\hline
\end{tabular}

En la tabla 9, que concierne a la variable especialidad, se evidencia que la significancia es mayor a .05. Por lo tanto, no existen diferencias significativas de cansancio emocional según especialidad.

Tabla 9

Estadísticos de prueba ANOVA para cansancio emocional según especialidad

\begin{tabular}{lccccc}
\hline & $\begin{array}{c}\text { Suma de } \\
\text { cuadrados }\end{array}$ & Gl & $\begin{array}{c}\text { Media } \\
\text { cuadrática }\end{array}$ & F & Sig. \\
\hline Entre grupos & 229.556 & 9 & 25.506 & .720 & .690 \\
Dentro de grupos & 8851.690 & 250 & 35.407 & & \\
Total & 9081.246 & 259 & & & \\
\hline
\end{tabular}

En la tabla 10, que concierne también a la variable especialidad, se evidencia que las significancias, salvo en despersonalización y realización personal, son mayores a .05. Por lo tanto, no existen diferencias significativas entre los componentes distorsión del entorno laboral y realización laboral según especialidad, y existen diferencias significativas en los componentes despersonalización y realización personal según especialidad. 
Tabla 10

Estadísticos de prueba Kruskal-Wallis para cuatro componentes del síndrome de burnout según especialidad

\begin{tabular}{lcccc}
\hline & $\begin{array}{c}\text { Desperso- } \\
\text { nalización }\end{array}$ & $\begin{array}{c}\text { Distorsión del } \\
\text { entorno laboral }\end{array}$ & $\begin{array}{c}\text { Realización } \\
\text { personal }\end{array}$ & $\begin{array}{c}\text { Realización } \\
\text { laboral }\end{array}$ \\
\hline Chi-cuadrado & 17.566 & 15.354 & 18.832 & 11.145 \\
Gl & 9 & 9 & 9 & 9 \\
Sig. asintótica & .041 & .082 & .027 & .266 \\
\hline
\end{tabular}

En la tabla 11, que concierne a la variable máximo nivel de estudios alcanzado, se evidencia que las significancias, salvo en realización personal y realización laboral, son mayores a .05. Por lo tanto, no existen diferencias significativas entre los componentes cansancio emocional, despersonalización y distorsión del entorno laboral según máximo nivel de estudios alcanzado, y existen diferencias significativas entre los componentes realización personal y realización laboral según máximo nivel de estudios alcanzado.

Tabla 11

Estadísticos de prueba Kruskal-Wallis para componentes del síndrome de burnout según máximo nivel de estudios alcanzado

\begin{tabular}{lccccc}
\hline & $\begin{array}{c}\text { Cansancio } \\
\text { emocional }\end{array}$ & $\begin{array}{c}\text { Despersona- } \\
\text { lización }\end{array}$ & $\begin{array}{c}\text { Distorsión } \\
\text { del entorno } \\
\text { laboral }\end{array}$ & $\begin{array}{c}\text { Realización } \\
\text { personal }\end{array}$ & $\begin{array}{c}\text { Realización } \\
\text { laboral }\end{array}$ \\
\hline Chi-cuadrado & 2.517 & 3.736 & .422 & 16.393 & 8.231 \\
Gl & 3 & 3 & 3 & 3 & 3 \\
Sig. asintótica & .472 & .291 & .936 & .001 & .041 \\
\hline
\end{tabular}

En la tabla 12, que concierne a la variable años de experiencia docente, se evidencia que la significancia es mayor a .05. Por lo tanto, no existen diferencias significativas de cansancio emocional según años de experiencia docente.

Tabla 12

Estadísticos de prueba ANOVA para cansancio emocional según años de experiencia docente

\begin{tabular}{lccccc}
\hline & Suma de cuadrados & Gl & Media cuadrática & F & Sig. \\
\hline Entre grupos & 78.859 & 5 & 15.772 & .445 & .817 \\
Dentro de grupos & 9002.387 & 254 & 35.442 & & \\
Total & 9081.246 & 259 & & & \\
\hline
\end{tabular}

En la tabla 13, que concierne también a la variable años de experiencia docente, se evidencia que todas las significancias, salvo en despersonalización, son menores a .05. Por lo 
tanto, no existen diferencias significativas de despersonalización según años de experiencia docente, y existen diferencias significativas entre los componentes distorsión del entorno laboral, realización personal y realización laboral según años de experiencia docente.

\section{Tabla 13}

Estadísticos de prueba Kruskal-Wallis para cuatro componentes del síndrome de burnout según años de experiencia docente

\begin{tabular}{lcccc}
\hline & $\begin{array}{c}\text { Despersona- } \\
\text { lización }\end{array}$ & $\begin{array}{c}\text { Distorsión del } \\
\text { entorno laboral }\end{array}$ & $\begin{array}{c}\text { Realización } \\
\text { personal }\end{array}$ & $\begin{array}{c}\text { Realización } \\
\text { laboral }\end{array}$ \\
\hline Chi-cuadrado & 8.977 & 11.480 & 27.640 & 18.527 \\
Gl & 5 & 5 & 5 & 5 \\
Sig. asintótica & .110 & .043 & .000 & .002 \\
\hline
\end{tabular}

En la tabla 14, que concierne a la variable turno de dictado, se evidencia que la significancia es menor a .05. Por lo tanto, existen diferencias significativas de cansancio emocional según turno de dictado.

Tabla 14

Estadísticos de prueba ANOVA para cansancio emocional según turno de dictado

\begin{tabular}{lccccc}
\hline & Suma de cuadrados & Gl & Media cuadrática & F & Sig. \\
\hline Entre grupos & 722.898 & 6 & 120.483 & 3.647 & .002 \\
Dentro de grupos & 8358.349 & 253 & 33.037 & & \\
Total & 9081.246 & 259 & & & \\
\hline
\end{tabular}

En la tabla 15, que concierne también a la variable turno de dictado, se evidencia que todas las significancias, salvo en distorsión del entorno laboral, son mayores a .05. Por lo tanto, no existen diferencias significativas entre los componentes despersonalización, realización personal y realización laboral según turno de dictado, y existen diferencias significativas de distorsión del entorno laboral según turno de dictado.

\section{Tabla 15}

Estadísticos de prueba Kruskal-Wallis para cuatro componentes del síndrome de burnout según turno de dictado

\begin{tabular}{lcccc}
\hline & $\begin{array}{c}\text { Despersonali- } \\
\text { zación }\end{array}$ & $\begin{array}{c}\text { Distorsión del } \\
\text { entorno laboral }\end{array}$ & $\begin{array}{c}\text { Realización } \\
\text { personal }\end{array}$ & $\begin{array}{c}\text { Realización } \\
\text { laboral }\end{array}$ \\
\hline Chi-cuadrado & 3.796 & 15.352 & 6.747 & 7.736 \\
Gl & 6 & 6 & 6 & 6 \\
Sig. asintótica & .704 & .018 & .345 & .258 \\
\hline
\end{tabular}




\section{Discusión}

A partir de los resultados, se puede afirmar que se cumplió parcialmente la primera hipótesis planteada, pues existen un nivel medio de síndrome de burnout según el análisis de cada uno de sus componentes: cansancio emocional, distorsión del entorno laboral, realización personal, realización laboral y despersonalización. No obstante, este último es el que ha presentado un puntaje muy cercano al alto. Ahora bien, tales resultados podrían deberse al nivel significativo de dispersión identificado en las respuestas de los profesores; de ahí, que no se pueda plantear afirmaciones concluyentes acerca de los síntomas de este trastorno presentes en la población. Sin embargo, sí es posible establecer algunas aseveraciones respecto de las diferencias existentes entre algunos de los componentes del síndrome de burnout según variables sociodemográficas, por lo que, en este aspecto, se cumple la segunda hipótesis, aunque también parcialmente por evidenciarse solo en ciertos componentes. A continuación, se explicará con detalle en qué componentes se evidencian diferencias significativas según las variables sociodemográficas identificadas.

Al realizar el análisis e interpretación de los datos según la variable sexo, se han identificado algunas diferencias significativas. Las mujeres evidencian mayores niveles de cansancio emocional respecto de los hombres, mientras que estos últimos se sienten más realizados laboralmente. Estos resultados concuerdan con los estudios realizados por Arias y Jiménez (2013) y Oliver (1993). Además, también coinciden con los trabajos efectuados por Asitimbay (2015), Manassero et al. (1995), Martínez (2015), Montejo (2014), Santiago, Otero- López, Castro y Villadefrancos (2008), Tifner et al. (2006) y Vidal (2013). Según la ideología sociocultural presente aún en nuestro contexto actual, a las mujeres se les enseña a ser más emotivas. Por ello, si experimentan sobrecarga laboral, lo exteriorizarán más que los hombres mediante alteraciones psicosomáticas y psicológicas.

La edad es una variable que también ha sido considerada en este estudio. Al respecto, se ha evidenciado que existen diferencias significativas de realización personal según edad. Así, los docentes cuyo rango de edad es entre 51 y 60 años evidenciarían una mayor realización personal respecto de los otros grupos etarios. Tal hallazgo se corresponde con los estudios de Manassero et al. (1995) y Ponce, Bulnes, Aliaga, Atalaya y Huerta (2014), quienes señalan que los docentes con mayor edad, lo que concuerda con su tiempo de servicio, probablemente, se sientan más realizados a nivel personal, debido a la estabilidad emocional y familiar que puedan estar experimentando.

Según Maslach y Jackson (1986), el estado civil es una variable que podría estar asociada a algunos componentes del burnout. Según los resultados de la presente investigación, existen diferencias significativas de realización personal según estado civil. De este modo, los docentes casados y divorciados son los que se sienten más realizados a nivel personal en comparación con los separados y convivientes. Tales hallazgos concuerdan con los propuestos por Silva et al. (2015). Ello podría deberse a que tanto los divorciados como los casados, debido al nivel de madurez que posiblemente hayan alcanzado, puedan haber adquirido estrategias de afrontamiento de problemas y, por ende, sentirse mejor consigo mismos. 
En cuanto al lugar de estudios de pregrado, los resultados evidencian que existen diferencias significativas de distorsión del entorno laboral según lugar de estudios de pregrado. Es decir, los profesores que estudiaron en una institución privada de educación superior suelen presentar un mayor nivel de distorsión de su entorno laboral en comparación con aquellos que estudiaron en una universidad estatal. Aunque no existen antecedentes de estudio al respecto, se ha podido establecer una relación entre tipo de institución de estudios de pregrado y el estrato socioeconómico. Así, según Ibáñez et al. (2012), los docentes que pertenecen a estratos más altos suelen sentirse más cansados emocionalmente y manifiestan mayores niveles de burnout. Esto podría relacionarse con los profesores que provienen de una universidad privada, quienes suelen evidenciar mayores problemas en el afrontamiento de problemas, lo que podría desencadenar en situaciones de distorsión de su entorno laboral y, de este modo, generar actitudes de rechazo hacia sus compañeros de trabajo e, incluso, hacia sus alumnos (Gil- Monte \& Peiró, 1997; Quiñones, 2012).

La especialidad del docente es una variable importante que, si bien no ha sido considerada de manera prioritaria en los antecedentes de estudio, podría estar asociada al desarrollo de algunos de los componentes del síndrome de burnout. Según el presente estudio, se ha identificado que existen diferencias significativas en los componentes despersonalización y realización personal según la especialidad del profesor. Así, los literatos son los que han manifestado mayores niveles de despersonalización, mientras que los antropólogos son los que evidenciaron una mayor realización personal. Estos resultados podrían explicarse a partir del estudio de Manassero et al. (1995), quienes, luego de realizar una investigación en una institución de Educación Básica Regular, concluyeron que los profesores que no han estudiado la carrera Educación y que, sin embargo, ejercen la docencia podrían evidenciar mayores síntomas de burnout si la tarea que realizan no se relaciona directamente con su profesión. Además, su nivel de realización personal se asocia con la percepción que demuestren respecto de qué tanto la labor pedagógica que han asumido les ayuda a desarrollarse profesionalmente o se vincule con su carrera. Entonces, el que los literatos no cumplan sus expectativas profesionales, dado que se encuentran dictando cursos básicos de Lenguaje en una universidad orientada a Ciencias, podría suscitar que evidencien mayores rasgos de despersonalización. Por el contrario, los antropólogos suelen dictar cursos de investigación, lo que podría estar más asociado con sus intereses académicos y profesionales, y ello podría estar contribuyendo al logro de su realización personal.

Otra variable asociada a los componentes de burnout es la relativa al máximo nivel de estudios alcanzado por los profesores. Al respecto, los resultados muestran que existen diferencias significativas entre los componentes realización laboral y realización personal. En la presente investigación, se identificó que los profesores con grado de doctor son los que se sienten más realizados, debido, probablemente, a los logros alcanzados y a que su retribución económica es mayor (Tifner et al., 2006).

Los años de experiencia docente constituyen otra variable asociada con la manifestación de algunos de los componentes de burnout. A partir de los datos hallados, se identificó que existen diferencias significativas entre los componentes distorsión del entorno laboral, realización personal y realización laboral según el tiempo de labor pedagógica. De esta manera, los profesores que han ejercido la docencia en menos 
tiempo (entre 1 y 5 años) son los que evidencian un nivel mayor de distorsión del entorno laboral. Por otro lado, los profesores más realizados a nivel personal son los que tienen entre 16 y 20 años de experiencia, mientras que los que están más realizados a nivel laboral presentan entre 26 y 30 años de ejercicio docente.

A pesar de que hay estudios como los de Boada et al. (2004), Díaz et al. (2010), Esteras et al. (2014) y Oliver (1993), en los que se indica que no hay diferencias significativas en esta variable de estudio, otros como los de Asitimbay (2015) y Manassero et al. (1995) manifiestan que sí podría establecerse ciertas diferencias. Así, según estos autores, los más jóvenes, es decir, los que recién se han insertado al mercado laboral, suelen ser más entusiastas, pero ese optimismo se pierde rápidamente cuando se enfrentan a obstáculos propios del sistema educativo y sus expectativas no logran materializarse. De este modo, este grupo estaría más propenso a padecer cansancio emocional, despersonalización y baja realización personal, lo que se corresponde con los datos hallados en el presente estudio. Por otra parte, los que evidencian más antigüedad en su experiencia docente son los que experimentan una mayor realización personal y laboral. De esta manera, los que están laborando entre 16 y 20 años, y posiblemente, tengan entre 38 y 45 años de edad, son los que, probablemente, hayan logrado una mayor estabilidad emocional y familiar, lo que podría generar que se sientan más realizados a nivel personal. Por otro lado, los que presentan una mayor antigüedad en su labor docente (entre 26 y 30 años), y tengan entre 48 y 55 años de edad, posiblemente, perciban que sus metas profesionales y laborales ya se han cumplido, lo cual podría explicar que se sientan más realizados a nivel laboral.

El turno de dictado es una variable que podría estar vinculada con la aparición de algunos componentes del burnout. A partir de los resultados, se puede afirmar que existen diferencias significativas de cansancio emocional y distorsión del entorno laboral según turno de dictado. Así, los profesores que manifiestan mayores niveles en tales componentes son los que dictan en dos turnos: tanto en la mañana, como en la tarde. Ello concuerda con el estudio realizado por Ibáñez et al. (2012). Tal circunstancia podría explicarse por el esfuerzo adicional que se genera al trabajar en horarios compuestos de muchos espacios "libres" que los docentes suelen utilizar para desarrollar otras actividades que también implican alguna labor intelectual.

\section{Conclusiones}

Es posible concluir que existen diferencias significativas entre algunos componentes del burnout según las variables sociodemográficas: sexo, edad, estado civil, lugar de estudios de pregrado, especialidad, máximo nivel de estudios alcanzado, años de experiencia y turno de dictado. A la luz de lo identificado mediante la investigación, es necesario realizar más investigaciones sobre el síndrome de burnout en docentes universitarios y sus variables sociodemográficas, tema que aún no ha sido estudiado ampliamente.

\section{Referencias}

Agudo, M. (2006). Burnout y engagement en profesores de primaria y secundaria. Fòrum de recerca, (12), 1-15. Recuperado de http://repositori.uji.es/xmlui/bitstream/ handle/10234/78668/forum_2006_34.pdf?sequence=1 
Arias, W. L., \& Jiménez, N. A. (2013). Síndrome de burnout en docentes de Educación Básica Regular de Arequipa. Educación, 22(42), 53-76. Recuperado de http://revistas. pucp.edu.pe/index.php/educacion/article/viewFile/5291/5288

Asitimbay, F. (2015). Agotamiento o sindrome de burnout en los docentes de bachillerato (Tesis de licenciatura, Universidad de Cuenca, Cuenca, Ecuador). Recuperado de http:// dspace.ucuenca.edu.ec/handle/123456789/23468

Benfica, A., \& Barbosa, I. (2015). Contribuições da Neurociência para a gestão de pessoas. Opción, 31(1), 113-133. Recuperado de http://www.produccioncientificaluz. org/index.php/opcion/article/view/20108

Boada, J., De Diego, R., \& Agulló, E. (2004). El burnout y las manifestaciones psicosomáticas como consecuentes del clima organizacional y de la motivación laboral. Psicothema, 16(1), 125-131. Recuperado de http://www.unioviedo.es/reunido/ index.php/PST/article/view/8198/8062

Cárdenas, M., Méndez, L. M., \& González, M. T. (2014). Evaluación del desempeño docente, estrés y burnout en profesores universitarios. Revista Electrónica Actualidades Investigativas en Educación, 14(1), 1-22. Recuperado de http://.redalyc.org/articulo. oa? id=44729876006

Cattaneo, M. R., \& Mandolesi, M. (2013). Síndrome de Burnout y engagement en docentes universitarios pertenecientes a la Universidad Nacional de Rosario. Relación con la vulnerabilidad psicosociolaboral (VPSL). Actas de Jornadas de Investigación, 93(3), 122-130. Recuperado de http://www.fpsico.unr.edu.ar/wp-content/uploads/2012/10/ TEXTOCOMPLETOCD1.pdf\#page $=122$

Díaz, F., López. A. M., \& Varela, M. T. (2010). Factores asociados al síndrome de burnout en docentes de colegios de la ciudad de Cali, Colombia. Universitas Psychologica, 11(1), 217-227. Recuperado de http://revistas.javeriana.edu.co/index.php/revpsycho/ article/view/649

Esteras, J., Chorot, P., \& Sandín, B. (2014). Predicción del burnout en los docentes: papel de los factores organizacionales, personales y sociodemográficos. Revista de Psicopatología y Psicología Clínica, 19(2), 79-92. Recuperado de http://revistas.uned. es/index.php/RPPC/article/view/13059

Farfán, M. E. (2009). Relación del clima laboral y síndrome de Burnout en docentes de educación secundaria en centros educativos estatales y particulares (Tesis de maestría, Universidad Nacional Mayor de San Marcos, Lima, Perú). Recuperado de http:// cybertesis.unmsm.edu.pe/bitstream/cybertesis/614/1/farfan_mm.pdf

Fernández, G., Ruiz, S., Toledo, V., \& Conchas, J. (2015). El síndrome de burnout y su relación con las creencias irracionales en el docente universitario. Revista EDUCATECONCIENCIA, 6(7), 22-38. Recuperado de http://www.tecnocientifica. com.mx/volumenes/V6N7A2.pdf

Gil-Monte, P. R., \& Peiró, J. M. (1997). Desgaste psíquico en el trabajo: El síndrome de quemarse. Madrid, España: Síntesis.

Gil-Monte, P. R., \& Peiró, J. M. (1999). Perspectivas teóricas y modelos interpretativos para el estudio del síndrome de quemarse por el trabajo. Anales de Psicología, 15(2), 261-268. Recuperado de http://www.um.es/analesps/v15/v15_2pdf/12v98_05Llag2.PDF

Hernández, R., Fernández, C., \& Baptista, P. (2014). Metodología de la Investigación. México, D.F.: McGraw-Hill. 
Ibáñez, J., López, J., Márquez, A., Sánchez, N., Flórez- Alarcón, L., \& Vera, A. (2012). Variables sociodemográficas relacionadas al síndrome de burnout en docentes de colegios distritales. Psychologia. Avances de la disciplina, 6(2), 103-116. Recuperado de http://www.redalyc.org/articulo.oa?id=297225788005

Jenaabadi, H., Azizi, B., Saeidi, F., Haghi, R., \& Hojatinasab, M. (2016). Relationship of Workaholism with Stress and Job Burnout of Elementary School Teachers. Health, 8(1), 1-8. Recuperado de http://www.scirp.org/journal/PaperInformation. aspx?paperID $=62560$

Manassero, M. A., Fornés, J., Fernández, M. C., Vázquez, A., \& Ferrer, V. A. (1995). Burnout en la enseñanza: análisis de su incidencia y factores determinantes. Revista de Educación, (308), 241-266. Recuperado de http://redined.mecd.gob.es/xmlui/ bitstream/handle/11162/70544/00820073003884.pdf?sequence=1

Martínez, J. P. (2015). Cómo se defiende el profesorado de secundaria del estrés: burnout y estrategias de afrontamiento. Journal of Work and Organizational Psychology, 31(1), 1-9. Recuperado de http://jwop.elsevier.es/es/como-se-defiende-el-profesorado/ articulo/S157659621500002X/\#.VrjEhlJhQXg

Maslach, C., \& Jackson, S. E. (1986). Maslach Burnout Inventory Manual (2. a Ed.). Palo Alto, California: Consulting Psychologists.

Maslach, C., Schaufeli W. B., \& Leiter M. P. (2001). Job Burnout. Annual Review of Psychology, 52, 397-422. doi: https://doi.org/10.1146/annurev.psych.52.1.397

Montejo, E. (2014). El síndrome del burnout en el profesorado de la ESO (Tesis doctoral, Universidad Complutense de Madrid, Madrid, España). Recuperado de http:// eprints.ucm.es/28333/

Oliver, C. (1993). Análisis de la problemática de estrés en el profesorado de enseñanzas medias: el burnout como síndrome específico (Tesis doctoral, Universidad Autónoma de Madrid, Madrid, España).

Ponce, C. R., Bulnes, M. S., Aliaga, J. R., Atalaya, M. C., \& Huerta, R. E. (2014). El síndrome del "quemado" por estrés laboral asistencial en grupos de docentes universitarios. Revista de investigación en psicología, 8(2), 87-112. Recuperado de http:// dialnet.unirioja.es/servlet/articulo? codigo $=2238169$

Quiñones, J. J. (2012). Burnout: eustrés y distrés en los maestros. Signo educativo, 21(211), 29-30.

Rabasa, B., Figueiredo-Ferraz, H., Gil-Monte, P. R., \& Llorca-Pellicer, M. (2016). El papel de la culpa en la relación entre el síndrome de quemarse por el trabajo y la inclinación al absentismo de profesores de enseñanza secundaria. Revista de Psicodidáctica, 21(1), 1-16. Recuperado de http://www.ehu.eus/ojs/index.php/psicodidactica/article/ viewFile/13076/13429

Sánchez, H., \& Reyes, C. (2015). Metodología y diseños en la investigación científica. (5a ed.). Lima, Perú: Business Support Aneth.

Santiago, M. J., Otero-López, J. M., Castro, C., \& Villadefrancos, E. (2008). Occupational stress in secondary school teachers: examining the role of students's disruptive behavior and/or others attitudes and the perceived difficulty in conflict management. European Journal of Education and Psychology, 1(1), 39-50. Recuperado de http://www. redalyc.org/pdf/1293/129318692003.pdf 
Silva, M. I., García, R. C., González, M., \& Ratto, A. (2015). Prevalencia del síndrome de quemarse por el trabajo y variables sociodemográficas en un grupo de maestras de Montevideo. Ciencias Psicológicas, 9(1), 55-62. Recuperado de http://www.scielo. edu.uy/scielo.php?script=sci_arttext\&pid=S1688-42212015000100006

Tenti, E. (2007). Consideraciones sociológicas sobre profesionalización docente. Educação $\mathcal{E}$ Sociedade, 28(99), 335-353. Recuperado de http://www.scielo.br/pdf/es/v28n99/ a03v2899.pdf

Tifner, S., Martín, P., Albanesi, S., \& De Bortoli, M. (2006). Burnout en el colectivo docente. Studium, (12), 279-291. Recuperado de http://dialnet.unirioja.es/descarga/ articulo/2542032.pdf

Vidal, F. (2013). Variables relacionadas con las dificultades en burnout en profesores y otros profesionales. (Tesis doctoral, Universidad de León, León, España). Recuperado de http://buleria.unileon.es/bitstream/handle/10612/2877/tesis_953539.PDF?sequence=1

Zaldívar, A., Estrada, R., Qui, S. O., Moncada, M., \& Aguilar, P. A. (2013). Relación entre Burnout y Desempeño Docente en el Nivel Superior. Revista Electrónica de Investigación en Educación Superior, 1(1), 1-8. Recuperado de https://www.researchgate. net/publication/251876057_Relacion_entre_Burnout_y_Desempeno_Docente_en_el_ Nivel_Superior

Fecha de recepción: 7 de febrero de 2017

Fecha de revisión: 15 de febrero de 2017

Fecha de aceptación: 17 de enero de 2018 
